\title{
Bounds on gravitational parity violation using a rotating torsion pendulum with chiral masses
}

\author{
Anaís Dorta-Urra ${ }^{1}$, Pedro Bargueño ${ }^{1, a}$ \\ ${ }^{1}$ Departamento de Física Aplicada, Universidad de Alicante, Campus de San Vicente del Raspeig, 03690 Alicante, Spain
}

Received: 22 February 2021 / Accepted: 27 June 2021 / Published online: 3 July 2021

(C) The Author(s) 2021

\begin{abstract}
In this letter we employ recent results on gravitationally induced parity violation with a rotating torsion pendulum whose test bodies are quartz enantiomers (Zhu et al. in Phys Rev Lett 121:261101, 2018) in order to estimate, using a simple model, Hari Dass's $\alpha_{2}$ constant which parametrizes the strength of parity violation in the gravitational interaction. The result here obtained, $\alpha_{2} \sim 10^{17}$, is in agreement with estimations based on high resolution experiments performed using chiral molecules, showing that the Hari-Dass's framework for spin-dependent gravity, together with our simple model, are versatile enough in order to be applied to the analysis of other experimental results involving spin-dependent gravitational effects. Interestingly, it can also be used to constrain indirectly parity-violating effects in macroscopic samples of quartz crystals due to electronnucleon interactions.
\end{abstract}

\section{Introduction}

Symmetries have played a protagonic role in the evolution of Physics. In particular, the importance of discrete symmetries within Quantum Field Theory can be summarized in the CPT theorem [1], which deeply links Lorentz invariance, the spinstatistics theorem and the conservation/violation of charge conjugation (C), parity (P) and time reversal (T). Moreover, the discovery of weak interaction effects in particle physics [2] paved the way for the study of left-right symetry breaking (P violation) in more complex systems such as nuclei [3] and atoms [4-6]. Interestingly, at present, $P$ violation effects in molecules have not been observed, although considerable efforts are being pursued by several groups, both in diatomic [7] and polyatomic systems, including chiral molecules [8]. Concerning these last ones, there is a long standing interest in the possible role played by $\mathrm{P}$ violation in chiral molecules and biological homochirality (see, for example, the recent

a e-mail: pedro.bargueno@ua.es (corresponding author) reviews $[9,10]$ and references therein), being this a very important open problem which could link Physics, Chemistry and Biology at a very fundamental level.

Regarding discrete symmetries, on the one hand, one of the main ingredients of the Standard Model of Particle Physics, which is one of the pillars of modern Physics, is P violation. On the other hand, General Relativity (GR), the other side of the coin, does not incorporate $\mathrm{P}$ violation although some of its extensions, with emphasis in Chern-Simons gravity (see [11] for a review), do. Interestingly, these and other P-odd gravitational effects could be tested in the near future by searching for amplitude birefringence in gravitational wave propagation $[11,12]$, for signals in the cosmic microwave background [13] and in the cosmological redshift [14], and by solar system observations [15], among others, which could serve to test some of these extensions to GR [16].

Apart from these astrophysical or cosmological tests of gravitational $\mathrm{P}$ violation, there have been Earth-based experiments trying mainly to test short- and long-range spindependent gravitational forces [17]. These gravitational Podd effects are of extraordinary importance because they break the equivalence principle, thus leading to a failure of GR [18]. In fact, the equivalence principle could be violated if new particles or interactions exist [19-23]. The best laboratory tests of the equivalence principle in terms of the Eötvös parameter, $\eta$, provide $\eta \sim 10^{-13}[24,25]$ but space missions give $\eta \sim 10^{-14}[26,27]$. Very recently, a new test of the equivalence principle designed to search for possible gravitational $\mathrm{P}$ violation using test bodies with different chiralities has been reported [28], giving $\eta \sim 10^{-13}$, and opening a new way to search for both P-odd gravitation and for a possible role played by a hypothetical gravitational $\mathrm{P}$-odd interaction in establishing molecular homochirality [10,29], whose origin remains as one of the most intriguing problems [30]. In addition, as pointed out by the authors of Ref. [28], these results could be used to set constraints on some parameters of P-odd gravitational potentials. 
The purpose of the present letter is, following the suggestion raised in [28], to obtain bounds on some models which incorporate long-range gravitational $\mathrm{P}$ violation in the weak field regime by using the recent experimental results reported in Ref. [28]. This manuscript is organized as follows: Sect. 2 introduces a modified Newtonian potential which incorporates P-odd effects, showing that it can be classified as a truly chiral influence [31] and comparing it with some other models which consider gravitational $\mathrm{P}$ violation, including some comments on bounds on gravitational $\mathrm{P}$ violation obtained using high resolution spectroscopic techniques in chiral molecules [32]. In Sect. 3 we estimate a new bound on gravitational $\mathrm{P}$ violation by using a rotating torsion pendulum which carries macroscopic chiral test masses and final remarks are left to Sect. 4.

\section{Parity-violating corrections to the Newtonian potential}

To the best of our knowledge, Leitner and Okubo were the first to inquire whether or not correlations between $\mathrm{P}$ violation in the weak interaction and its corresponding weakness could be extended to gravitation, proposing a parametrization to the Newtonian potential which includes terms which violate $\mathrm{C}, \mathrm{P}$ and $\mathrm{T}$ symmetries [33]. Some years after that, Hari Dass introduced [34-36] a different (but also phenomenological) parametrization which, assuming CPT conservation, included $\mathrm{P}$ and $\mathrm{T}, \mathrm{C}$ and $\mathrm{P}$ and $\mathrm{C}$ and $\mathrm{T}$ violation in an extended Newtonian potential.

Under weak field conditions, let us assume that test particles are nonrelativistic. Then, maintaining only terms in the interaction energy that are linear in the expectation value of the particle spin, $\vec{s}$, and that are consistent with rotational and translational invariance and the dimensionality of the (only) gravitational coupling constant, we arrive at $(c=1)$ [36]

$U(r)=G M\left(\alpha_{1} \frac{\vec{s} \cdot \vec{r}}{r^{3}}+\alpha_{2} \frac{\vec{s} \cdot \vec{v}}{r^{2}}+\alpha_{3} \frac{\vec{s} \cdot(\vec{r} \times \vec{v})}{r^{3}}\right)$,

where $\vec{v}$ is the expectation value of the particle relative to a mass $M$ which sources the gravitational field (the Earth in our case), being $\vec{r}$ the expectation value of the relative position of this particle. The $\alpha_{i}$ dimensionless constants parametrize the strength of discrete symmetry violations.

Let us remark that the nature of the couplings of the spin to gravitational fields is of extraordinary importance to our understanding of the microscopical structure of gravity. In particular, it is our interest to fix our attention in the $\alpha_{2}$ term, which gives rise to gravitational $\mathrm{P}$ violation and also to a violation of the equivalence principle [54]. Even more, this term is the only one which constitutes, using Barron's widely accepted definitions [31] a truly chiral influence: true chiral- ity is exhibited by systems that exist in two distinct enantiomeric states that are interconverted by space inversion but not by $\mathrm{T}$ reversal combined with any proper spatial rotation [31]. Therefore, following Barron, the $\vec{s} \cdot \vec{v}$ term can lift the degeneracy between objects of different chirality. On the contrary, the $\alpha_{1}$ term, is P- and T-odd but PT-even and, therefore, is classified as a falsely chiral influence. An interesting example of a falsely chiral influence is the axion-mediated electron-nucleon interaction [37,38].

We would like to note that, from the experimental side, upper bounds on $\alpha_{2}$ have been estimated both from astrophysical measurements [39-41] $\left(\alpha_{2}<10^{21}\right)$ and from experiments with chiral molecules [32] $\left(\alpha_{2}<10^{17}\right)$ and ultracold neutrons [43] $\left(\alpha_{2}<3 \cdot 10^{10}\right)$.

Finally, in order to put some bounds on P-odd gravity, let us remark that the key point is to note that there is a $\mathrm{P}$ violating energy difference (PVED) between objects with different chirality (enantiomers) due to the $\alpha_{2}$ term of the gravitational potential. Specifically, the P-odd gravitational energy difference between left (L) and right (R) molecular enantiomers is given by [32]

$$
\begin{aligned}
\Delta E_{\alpha_{2}}^{\mathrm{PV}} & =\left|E_{\alpha_{2}, L}^{\mathrm{PV}}-E_{\alpha_{2}, R}^{\mathrm{PV}}\right|=2 E_{\alpha_{2}, L}^{\mathrm{PV}}=2 E_{\alpha_{2}, R}^{\mathrm{PV}} \\
& =\alpha_{2} \frac{G M}{r^{2} m_{e}} \sum_{i} \chi\left(x_{i}\right),
\end{aligned}
$$

where $m_{e}$ stands for the mass of the electron and $\chi\left(x_{i}\right)$ refers to its chirality, defined as [44]

$$
\begin{aligned}
\chi(x)= & N_{e} \sum_{s} \int d^{3} x_{2} \ldots d^{3} x_{N} \psi^{\dagger}\left(x, x_{2}, \ldots, x_{N}, s_{1}, \ldots s_{N}\right) \\
& \times\left(\vec{s}_{e} \cdot \vec{p}_{e}\right) \psi\left(x, x_{2}, \ldots, x_{N}, s_{1}, \ldots s_{N}\right)
\end{aligned}
$$

where $N_{e}$ is the total number of electrons of the sample and $\vec{p}_{e}$ their linear momentum, $\psi$ being the many-electron wave function with electron spatial coordinates $x_{i}$ and spin coordinates $s_{i}$. It is important to note that in the latter equation, the corresponding electronic chirality has to be computed at each point of the sample.

As a side remark we note that, in addition to this phenomenological P-odd long range gravitational potential, there are some models beyond GR which include the coupling of fermionic degrees of freedom to gravity in the presence of a non-vanishing torsion. Interestingly, these models give place to P-odd contact potentials which, in the nonrelativistic limit, involve also the electron helicity, as in the electroweak case [45], but this time the coupling constant turns out to be $G$ instead of Fermi's constant, $G_{F}$. Specifically, a non-minimal fermionic coupling to gravity in presence of torsion give rise to an effective weak charge given by [46] $Q_{G}=-9 \sqrt{2} \beta(Z+N) \frac{G}{G_{F}}$, where $Z$ and $N$ are the number of protons and nucleons of the sample, respectively, $\beta=\frac{2 \gamma \alpha}{\gamma^{2}+1}$ being $\gamma$ the Barbero-Immirzi parameter. The parameter $\alpha \neq 0$ implies the aforementioned non-minimal 
coupling [46]. Concerning the experimental detectability of this effect, atomic $\mathrm{P}$ violation experiments show an experimental constraint on $\beta$ which is $\beta<10^{30}$ [47]. In addition, non-conclusive searches for the PVED between the two enantiomers of the bromochlorofluoromethane molecule have been used to obtain $\beta<10^{16}$ [32] which is, to the best of our knowledge, the most stringest bound on P-odd contact gravity.

\section{Bounds on $\alpha_{2}$}

The key point in order to obtain stringent bounds on gravitational P violation is to work with a macroscopic sample of chiral electrons due to the linear growing of the electron chirality with $N_{e}, \chi \sim N_{e}$. The importance of $N_{e}$ can be shown, for instance, by comparing Heckel's et al. results on P-odd long range interactions (non-necessarily linked with gravity) using a torsion pendulum with $N_{e} \sim 9 \cdot 10^{22}$ and those by Bargueño et al. considering $N_{e} \sim 10^{2}$ for a single bromochlorofluoromethane molecule. In the first case, a hypothetical dimensionless P-odd coupling constant is $\sim 10^{-57}$ while the second approach leads to $\sim 10^{-4}$ [32].

In this sense, Zhu's et al. recent experimental results concerning gravitational $\mathrm{P}$ violation turn out to be essential. In Ref. [28], the authors report the first measurements of P-odd gravity using two enantiomeric quartz crystals as their test masses, obtaining that the difference in the acceleration of left- and right-handed crystals towards Earth is $\Delta a=-1.7 \pm 4.1$ (stat) \pm 4.4 (syst) $10^{-15} \mathrm{~m} \mathrm{~s}^{-2}$ (1- $\sigma$ statistical uncertainty) and thus paving the way for the search for possible P-odd effects in gravity.

In order to estimate some bounds on $\alpha_{2}$ using Zhu's et $a l$. recent results, let us make the following approximations. First, the total number of electrons of the sample is estimated as follows: the two chiral quartz crystals $\left(\mathrm{SiO}_{2}\right)$ are machined into $1.5 \mathrm{~cm}^{3}$ cubes [28]. Therefore, considering that the average molecular size is $\sim 1 \AA$, the pendulum contains $\sim 10^{24}$ molecules. As the number of electrons for a single $\mathrm{SiO}_{2}$ molecule is 30 , the total number of electrons contained in the pendulum is $N_{e} \sim 10^{25}$. At this point we make an important approximation: all of these $10^{25}$ electrons are chiral. We base this assertion on the basis that P-odd effects have been computed in quartz showing that contact electroweak P-odd interactions, which are based on the electron helicity operator, produce an energy splitting between different quartz enantiomers [48-50]. Second, in order to calculate $\Delta E_{\alpha_{2}}^{\mathrm{PV}}$, we employ the same approximation we used in Ref. [32] concerning the electron chirality. Although this quantity can be calculated using very accurate $a b$ initio methods, a rough estimate can be made by taking
Table 1 Experimental bounds for the $\alpha_{2}$ parameter. See text for details

\begin{tabular}{lll}
\hline$\alpha_{2}$ & References & Method \\
\hline$\leq 10^{21}$ & {$[39-41]$} & Astrophysical measurements \\
$\leq 10^{17}$ & {$[32]$} & Spectroscopy with chiral molecules \\
$\leq 10^{10}$ & {$[43]$} & Ultracold neutrons \\
$\sim 10^{17}$ & This analysis & Chiral torsion pendulum \\
\hline
\end{tabular}

$\Delta E_{\alpha_{2}}^{\mathrm{PV}} \sim \alpha_{2} \frac{G M_{\oplus} N_{e}}{R_{\oplus}^{2} c^{2}} \hbar v_{\oplus} \sim 10^{-31} N_{e} \alpha_{2} v_{\oplus} c^{-1}(\mathrm{eV})$,

where the constants $c$ and $\hbar$ have been explicitly introduced, $R_{\oplus}$ and $M_{\oplus}$ stands, respectively, for the radius and mass of the Earth and $\left\langle v_{e}\right\rangle$ is the surface velocity of the Earth, as we revolve about the center. And third, by considering that the difference in the energy of left- and right-handed crystals is roughly that of the equivalence-principle violating torque, $\tau$, appearing in the pendulum (here we consider $\tau$ as the only characteristic gravitational P-odd energy scale), we have that $\Delta E_{\alpha_{2}}^{\mathrm{PV}} \sim \tau$. Then, after considering that $\tau \sim p_{c} \Delta a$, $p_{c}=63.2 \mathrm{~g} \mathrm{~cm}$ being the mass moment of the pendulum [28], we obtain

$\alpha_{2} \sim \frac{10^{32} c}{N_{e}\left\langle v_{e}\right\rangle}$.

Taking a crude estimation of the surface velocity of the Earth as $v_{\oplus} \sim 10^{-10} c$ and remembering that $N_{e} \sim 10^{25}$, we get

$\alpha_{2} \sim 10^{17}$.

At this point a couple of comments are in order. (i) The estimation [55] here obtained has to be taken with caution due to the fact that we have considered that all the electrons of the sample are chiral, while a better approximation can be done by considering that only the electrons situated in external layers of the molecule, which are more delocalized, are chiral. This is linked with the crude estimation of the chirality of the sample, which is one of the key points. In this sense, due to the overestimation of the chirality, Eq. (6) might be taken as a lower bound; (ii) the result here obtained for $\alpha_{2}$ coincides with that estimated in Ref. [32] using spectroscopic results of a single bromochlorofluoromethane molecule but is still far from that found using experiments with ultracold neutrons [43] (see Table 1).

\section{Final remarks}

On the one hand, from the previous discussion in Sect. 3, we think that it would be difficult to obtain tighter bounds on $\alpha_{2}$ using the kind of experiments reported in [28], although working with a large number of chiral electrons or improving some technical features of the experimental setup might be 
useful, as commented in [28]. However, as we have employed a very simplified theoretical model in order to calculate both the electron chirality and the number of chiral electrons of the sample, we think that there is still room for improvement by using high level ab initio techniques or more sophisticated models such as those presented in [50]. In addition, the use of other macroscopic sources whose electroweak P violating effects are well studied, such as the Chiral Oxorhenium(V) Complexe [51], for example, could serve to improve the current bounds on gravitational $\mathrm{P}$ violation.

On the other hand, note that electroweak effects are of fundamental importance because, if gravity does not conserve $P$, then it would couple differently to left and right handed quartz crystals. Very importantly, the property of these crystals that is being tested is exactly the electron chirality of the sample. Therefore, as this quantity is non-zero due to the the electroweak interaction between electrons and nuclei of quartz, in order for gravity to couple in a different way to leftand right-handed crystals there should be a P-odd factor in the gravitational interaction. From a phenomenological point of view, Hari Dass's potential incorporates it and, therefore, their effects could serve as an indirect confirmation of electroweak effects inside quartz, whose effects in establishing homochirality on Earth are still under debate [52,53].

Finally, we would like to remark that the estimation here obtained for $\alpha_{2}$ employing using recent experimental results provided by a chiral torsion pendulum [28] at 1- $\sigma$ statistical uncertainty using the Earth as the source is compatible with that obtained using ultracold neutrons at a $95 \%$ CL using the Sun as the source [43]. In this sense, we think that the Hari-Dass's phenomenological description for spindependent gravity [34-36], together with the simple model we have presented, are versatile enough in order to be applied to the analysis of other experimental results involving spindependent gravitational interactions.

Acknowledgements P. B. is funded by the Beatriz Galindo contract Ministerio de Educación y Formación Profesional BEAGAL 18/00207, Spain. This work is dedicated to Ana, Inés and Lucía.

Data Availability Statement This manuscript has no associated data or the data will not be deposited. [Authors' comment: Data sharing not applicable. This article describes entirely theoretical research.]

Open Access This article is licensed under a Creative Commons Attribution 4.0 International License, which permits use, sharing, adaptation, distribution and reproduction in any medium or format, as long as you give appropriate credit to the original author(s) and the source, provide a link to the Creative Commons licence, and indicate if changes were made. The images or other third party material in this article are included in the article's Creative Commons licence, unless indicated otherwise in a credit line to the material. If material is not included in the article's Creative Commons licence and your intended use is not permitted by statutory regulation or exceeds the permitted use, you will need to obtain permission directly from the copyright holder. To view a copy of this licence, visit http://creativecomm ons.org/licenses/by/4.0/.

Funded by SCOAP ${ }^{3}$.

\section{References}

1. R.F. Streater, A.S. Wightman, PCT, Spin and Statistics, and All That (Princeton University Press, Princeton, 1964)

2. T.D. Lee, C.N. Yang, Phys. Rev. 104, 254 (1956)

3. C.S. Wu et al., Phys. Rev. 105, 1413 (1957)

4. M.A. Bouchiat, C. Bouchiat, J. Phys. (France) 35, 899 (1974)

5. I.B. Khriplovich, Parity Nonconservation in Atomic Phenomena (Gordon and Breach, London, 1991)

6. C.S. Wood et al., Science 275, 1759 (1997)

7. D. DeMille et al., Phys. Rev. Lett. 100, 023003 (2008)

8. P. Schwerdtfeger, The search for parity violation in chiral molecules, in Computational Spectroscopy, ed. by J. Grunenberg (Wiley-VCH, Weinheim, 2010), pp. 201-221

9. V.A. Davankov, Symmetry 10, 749 (2018)

10. A. Dorta-Urra, P. Bargueño, Symmetry 11, 661 (2019)

11. S. Alexander, N. Yunes, Phys. Rep. 480, 1 (2009)

12. R. Jackiw, S.Y. Pi, Phys. Rev. D 68, 104012 (2003)

13. A. Lue, L. Wang, M. Kamionkowski, Phys. Rev. Lett. 83, 1506 (1999)

14. B. Altschul, M. Mewes, Phys. Rev. D 84, 083512 (2011)

15. Y. Nakamura et al., Class. Quantum Gravity 36, 105006 (2019)

16. E. Berti et al., Class. Quantum Gravity 32, 243001 (2015)

17. M.S. Safronovait et al., Rev. Mod. Phys. 90, 025008 (2018)

18. C.M. Will, Living Rev. Relativ. 17, 4 (2014)

19. E. Fischbach et al., Phys. Rev. Lett. 56, 1427 (1986)

20. T. Damour, Class. Quantum Gravity 29, 184001 (2012)

21. P. Fayet, Phys. Rev. D 97, 055039 (2018)

22. P.W. Graham et al., Phys. Rev. D 93, 075029 (2016)

23. S.M. Carroll, S. Mantry, M.J. Ramsey-Musolf, C.W. Stubbs, Phys. Rev. Lett. 103, 011301 (2009)

24. S. Schlamminger et al., Phys. Rev. Lett. 100, 041101 (2008)

25. T.A. Wagner, S. Schlamminger, J.H. Gundlach, E.G. Adelberger, Class. Quantum Gravity 29, 184002 (2012)

26. P. Touboul et al., Phys. Rev. Lett. 119, 231101 (2017)

27. F. Hofmann, J. Müller, Class. Quantum Gravity 35, 035015 (2018)

28. L. Zhu et al., Phys. Rev. Lett. 121, 261101 (2018)

29. P. Bargueño, Chirality 27, 375 (2015)

30. N. Jones, Nature (London) 481, 14 (2012)

31. L.D. Barron, Chem. Phys. Lett. 123, 423 (1986)

32. P. Bargueño, R.P. de Tudela, Phys. Rev. D 78, 102004 (2008)

33. J. Leitner, S. Okubo, Phys. Rev. B 136, 1542 (1964)

34. N.D. Hari Dass, Phys. Rev. Lett. 36, 393 (1976)

35. N.D. Hari Dass, Gen. Relativ. Gravit. 8, 89 (1977)

36. N.D. Hari Dass, Ann. Phys. (N.Y.) 107, 337 (1977)

37. J.E. Moody, F. Wilczek, Phys. Rev. D 30, 130 (1984)

38. L.D. Barron, Chirality 24, 957 (2012)

39. L.D. Almeida, G.E.A. Matsas, A.A. Natale, Phys. Rev. D 39, 677 (1989)

40. J.M. LoSecco et al., Phys. Lett. A 138, 5 (1989)

41. J.R. Klein, S.E. Thorsett, Phys. Lett. A 145, 79 (1990)

42. A.K. Mann, H. Primakoff, Orig. Life 11(3), 255 (1981)

43. I. Altarev et al., Phys. Rev. Lett. 103, 081602 (2008)

44. R.A. Hegstrom, J. Mol. Struct. (Theochem) 232, 17 (1991)

45. R. Berger, in Relativistic Electronic Structure Theory, Part 2, applications, ed. by P. Schwerdtfeger (Elsevier, Amsterdam, 2004), p. 188

46. L. Freidel, D. Minic, T. Takeuchi, Phys. Rev. D 72, 104002 (2005)

47. S.C. Bennett, C.E. Wieman, Phys. Rev. Lett. 82, 2484 (1999)

48. G.E. Tranter, Nature 318, 172 (1985)

49. A.J. MacDermott, G.E. Tranter, Croat. Chem. Acta 62, 165 (1989) 
50. A.J. MacDermott, G.E. Tranter, Chem. Phys. Lett. 163, 1 (1989)

51. F. De Montigny et al., Phys. Chem. Chem. Phys. 12, 8792 (2010)

52. E.I. Klabunovskii, Astrobiology 1, 127 (2001)

53. A.J. MacDermott, in Comprehensive Chirality, vol. 8, ed. by E.M. Carreira, H. Yamamoto (Elsevier, 2012), p. 11-38

54. In fact, in all metric theories of gravitation we have $\alpha_{1}=\alpha_{2}=0$
55. Interestingly, Mann and Primakoff [42] estimated also by an-orderof-magnitude calculation the effect of the interaction of electrons having non-zero chirality with racemic mixtures of amino acids, which is one of the mechanisms considered to explain molecular homochirality 